\title{
Book Review „Die Entdeckung des Nomos - Carl Schmitt und die Geographie“
}

\author{
J. Prinz and C. Schetter \\ Universität Bonn, Bonner Asienzentrum, Zentrum für Entwicklungsforschung, Department of Political and \\ Cultural Change, Walter-Flex-Str. 3, 53113 Bonn, Germany \\ Correspondence to: C. Schetter (c.schetter@uni-bonn.de)
}

Legg, Stephen: Spatiality, Sovereignty and Carl Schmitt. Geographies of the Nomos, London, Routledge $\mathcal{G}$ Chapman and Hall, 306 pp., ISBN-13: 978-0-415-52286-1, € 32.99, 2011.

Das Interesse am Werk von Carl Schmitt (1888-1985) hat im vergangenen Jahrzehnt stark zugenommen. Fand Carl Schmitt in den großen politischen Debatten des Kalten Krieges - nicht zuletzt aufgrund seiner aktiven Rolle im Nationalsozialismus - überhaupt kein Gehör, so wird jüngst in ganz unterschiedlichen Strängen politischer Debatten verstärkt auf ihn Bezug genommen. Ausgehend von der Frage nach der neuen globalen Ordnung gewannen die Thesen Carl Schmitts in Debatten um eine multipolare Weltordnung genauso an Gewicht wie in der Diskussion über die angebliche Konfrontation zwischen ,westlicher" und ,islamischer“ Welt nach 9/11 oder in Schriften zur „Entpolitisierung" liberal-demokratischer Systeme. Während sich dieses Interesse zunächst vornehmlich auf Politikwissenschaftler und Philosophen konzentrierte, die sich mit Schmitts Kritik am Parlamentarismus, seiner Freund-Feind-Unterscheidung, seinem Verständnis des Ausnahmezustands oder seiner Rolle im Nationalsozialismus befassten, erscheint Carl Schmitt heute in politischen Debatten nahezu allgegenwärtig - und selbst bei der populären US-amerikanischen Fernsehserie „24“, die sich mit Anti-Terror-Einsätzen beschäftigt, könnte man meinen, dass Carl Schmitt am Drehbuch mitgeschrieben hat. In dieser Serie steht Schmitts Verständnis von Politik als heroische Entscheidungsfindung einzelner Personen unter Hinwegsetzung über gesellschaftliche und verfassungsrechtliche Normen im Vordergrund. Erwähnenswert ist zudem, dass Schmitt, der wohl mit Fug und Recht als erzkonservativ, bellezistisch und anti-demokratisch charakteri- siert werden kann, zuletzt verstärkt von ,linken“ Autoren ins Feld geführt wird. So sind es beispielsweise populäre ,linke“ Autoren wie Chantal Mouffe (in „,On the Political“ oder in ,The Democratic Paradox“) und Giorgio Agamben (,Homo Sacer"; „State of Exception“), die in ihren theoretischen Ansätzen explizit auf Ideen von Carl Schmitt Bezug rekurrieren.

Es überrascht, dass im Zuge dieser Schmitt-Euphorie erst in den letzten Jahren Schmitts Raumdenken näher untersucht wurde, u.a. im vorliegenden Band, der vor allem in Großbritannien und in den USA tätige Geographen zusammenbringt. So ist es erstaunlich, dass Schmitt zwar in Politikwissenschaft und Philosophie bereits stark rezipiert wird, in der Geographie bislang aber wenig Beachtung fand. Dabei durchzieht die Arbeiten von Schmitt spätestens seit den 1930er Jahren bis zu seinem Spätwerk ,Theorie des Partisanen“ (1963) eine eigene Raumphilosophie, in deren Mittelpunkt der Begriff des ,Nomos“ steht. Um die Bedeutung dieser Raumphilosophie für Schmitts Gesamtwerk zu unterstreichen, sei hier die nicht gerade waghalsige These angeführt, dass die Dialektik zwischen dem Politischen und Raum den roten Faden im Werk von Carl Schmitt darstellt.

Die wenigen vorliegenden Annäherungen an Schmitts Raumdenken fanden bislang weniger über eine direkte Auseinandersetzung mit Schmitt statt, als über die Rezeption populärer Autoren wie Agamben, Mouffe, Laclau oder Žižek; hiervon geben auch die längeren Aufsätze im vorliegenden Sammelband ,Spatiality, Sovereignty and Carl Schmitt“ Zeugnis. So nährt sich etwa Rory Rowan über Mouffes Kommentar zur multipolaren Ordnung der Welt an Carl Schmitt an, während Mathew Coleman sich über Agambens ,homo sacer“ Schmitts Freund-Feind-Abgrenzung annimmt. Timothy W. Luke wiederum betrachtet Schmitt aus 
der Perspektive von 9/11 und Samuel Huntingtons „Clash of Civilizations“, den er als verwandtes Projekt interpretiert. Vereint werden diese unterschiedlichen Zugriffe auf das Werk von Carl Schmitt in diesem Band dadurch, dass sie sich in der ein oder anderen Weise alle mit Einzelaspekten der Schmitt'schen Raumphilosophie beschäftigen und so einen Eindruck von der interpretativen Fläche vermitteln, die Schmitts Werk eröffnet.

Angesichts der bisherigen Vernachlässigung von Schmitts Raumphilosophie strebt der vorliegende Band an, ein umfassendes Verständnis von Schmitts Raumphilosophie und ihrem Verhältnis zu seinen anderen Werken zu erarbeiten. Das Ziel wird ob eines Mangels an Systematik allerdings nur teilweise erreicht. Zwar bietet der Band gerade für die englischsprachige Leserschaft eine Einführung in die Diskussion um Schmitts Raumphilosophie, u.a. durch am Anfang des Bandes zum ersten Mal in englischer Übersetzung vorliegende Schmitt-Aufsätze „Völkerrechtliche Formen des modernen Imperialismus“ (1933) und „Großraum gegen Universalismus" (1939) zur Monroe-Doktrin sowie durch Stuart Eldens Aufsatz „Reading Schmitt Geopolitically: Nomos, Territory and Großraum “. Die für die deutschsprachige Leserschaft interessanteren Aufsätze, die sich intensiver mit Schmitts „Nomos" befassen, stehen allerdings eher nebeneinander als gemeinsam zum Verständnis von Schmitts „Nomos“ beizutragen, wozu die Begutachtung von Schmitts Schriften durch die Linse Dritter häufig beiträgt.

Zudem überwiegt der Eindruck, dass die Auseinandersetzung mit den Konturen von Schmitts Raumdenken unter dem Ziel steht, es mit Schmitts Verständnis des Politischen bzw. anderen Aspekten seines Werkes in Verbindung zu setzen. In diesem Zusammenhang rückt auch die Auswahl der zu anfangs abgedruckten Übersetzungen in ein anderes Licht. So analysiert Daniel Clayton die Spannung zwischen Schmitts „Nomos“Begriff und seinem Begriff des Politischen und führt die Figur des Partisanen als Beispiel an. Claudia Minca geht Schmitts ,Nomos“-Begriff unter Betrachtung der „Naturalisierung der Bindung der Menschen an den Raum“ als Ausgangspunkt für gegenwärtig bedeutsame Diskurse um „Biopolitik“ nach. Thalin Zarmanian befasst sich mit dem Verhältnis zwischen den Begriffen der „Ortung“ und „Ordnung“" und präsentiert den „Nomos“-Begriff als Schmitts Versuch, Krieg einzudämmen.

Die grundsätzlich hilfreichen und detailreichen, wenn auch selektiven Kurzaufsätze zu einzelnen Schlüsselbegriffen aus Schmitts Raumphilosophie, etwa zu seinem Verständnis von „Liniendenken“ und sogenannten „Freundschaftslinien“ (amity lines) (Stirk), füllen eine Lücke in der Literatur, stehen aber ebenfalls kaum in Zusammenhang mit den anderen Beiträgen. Diese Problematik wird dadurch verstärkt, dass das Buch für den Leser, der erstmals mit Schmitt in Berührung kommt, verwirrend und überkomplex wirken mag. So handelt es sich gewiss um keine leichte Lektüre und bedarf einiger intellektueller Kraftanstrengungen. So hätte dem Buch in der durchaus sehr lesenswer- ten Einleitung von Stephen Legg gut getan, wenn hier eine Einführung in das Werk von Schmitt angeboten und in die unterschiedlichen historischen Phasen seines Raumdenkens eingeführt worden wäre.

Die Auseinandersetzung mit Schmitts Raumverständnis in diesem Buch offenbart zudem durchaus interessante Kontroversen. Während etwa Stuart Elden Carl Schmitt als einen klassischen „Geopolitiker“ bezeichnet, hält hier Claudio Minca dagegen, dass sich Schmitt doch eher mit einer räumlichen Ontologie beschäftigte und ihn gerade eine Verbindung dieser Ontologie mit konkreten physischen Räumen wenig interessiert habe. In ähnlicher Weise merkt man diesem Sammelband das Wechselbad der Gefühle der Autoren an. So fühlen sich viele Autoren gleichsam von Schmitt angezogen und abgestoßen - ein Phänomen, das häufig in Schmitt-Rezeptionen vorzufinden ist, wie etwa auch in dem Sammelband „The Internation Political Thought of Carl Schmitt“ (2007), herausgegeben von Louiza Odysseos und Fabio Petito, der die Diskussion zu Schmitt im Bereich der Internationalen Beziehungen aufgearbeitet hat.

Alles in allem handelt es sich um eine in der Geographie längst überfällige Auseinandersetzung mit Carl Schmitt. Das Buch offenbart eine willkommene Zusammenschau von Schlaglichtern, die auf das umstrittene Werk von Carl Schmitt geworfen werden und das Potential besitzen, Schmitts Raumphilosophie mit gegenwärtigen Fragen von Geopolitik und politischer Geographie in Verbindung zu bringen. Für die deutschsprachige Schmitt-Rezeption bietet es eine neue Perspektive, die als Inspiration für zukünftige Arbeiten dienen kann. Jeweils für sich genommen bieten die Beiträge originelle Zugänge zu Schmitts Raumdenken und spiegeln die Vielfalt der internationalen und disziplinären Schmitt-Rezeptionen wider. Der Sammelband macht zudem deutlich, dass die Diskussion über die Raumphilosophie Carl Schmitts in der Disziplin der Geographie erst begonnen hat; so ist damit zu rechnen, dass in den kommenden Jahren gerade in der theoretischen und politischen Geographie die wissenschaftlichen Auseinandersetzungen mit Carl Schmitts Werk zunehmen werden.

\section{Literatur}

Agamben, G.: Homo Sacer, Frankfurt a.M., 2002.

Agamben, G.: Ausnahmezustand, Frankfurt a.M., 2004.

Huntington, S.: Kampf der Kulturen. Die Neugestaltung der Weltpolitik im 21. Jahrhundert, Wien, 1996.

Mouffe, C.: Über das Politische: Wider die kosmopolitische Illusion, Frankfurt, 2007.

Mouffe, C.: Das demokratische Paradox, Wien u. Berlin, 2010.

Odysseos, L. and Petito, F.: The International Political Thought of Carl Schmitt. Terror, Liberal War and the Crisis of Global Order, London, 2007.

Schmitt, C.: Der Begriff des Politischen, Berlin, 1932/2002.

Schmitt, C.: Völkerrechtliche Großraumordnung mit Interventionsverbot für raumfremde Mächte, Berlin, 1941/1991. 
Schmitt, C.: Der Nomos der Erde, Berlin, 1950/1997.

Schmitt, C.: Theorie des Partisanen. Zwischenbemerkung zum Begriff des Politischen, Berlin, 1963/2006. 